\title{
Short-term results of the Warden technique: a retrospective single-center study
}

\author{
G Liguori, , T Camargo, D Akerman, J Penha, L Miana, L Caneo, C Tanamati, M Jatene \\ From 23rd World Congress of the World Society of Cardio-Thoracic Surgeons \\ Split, Croatia. 12-15 September 2013
}

\section{Background}

The Warden technique was introduced in an attempt to decrease the incidence of sinus node dysfunction and venous obstruction after the repair of partial anomalous pulmonary venous connection to the superior vena cava. In this study, we sought to report and evaluate the immediate outcomes of our experience with the Warden technique.

\section{Methods}

We conducted a retrospective study of 9 patients with anomalous drainage of the pulmonary veins to superior vena cava who have undergone the Warden technique during the year of 2011. We analyzed medical records, surgical reports and results of complementary tests.

\section{Results}

Besides the anomalous pulmonary venous connection to the superior vena cava, 5 (56\%) patients also presented an ostium secundum or patent foramen ovale atrial defect and $2(22 \%)$ presented persistent left superior vena cava. One patient presented Turner Syndrome. Before surgery, 3 (33\%) patients presented some form of conduction disorder: two right bundle branch block and one atrial extrasystole. The mean age at surgery was $10.8 \pm 7.6(\min =2$, $\max =29.7)$ years, and the distribution of the sexes was $2: 1$, the majority being male. None patient underwent reoperation or evolved to death during the follow-up period. After surgical repair, a forth patient presented with right bundle branch block. The degree of right chambers dilatation improved significantly at both atrial $(\mathrm{p}=0,002)$ and ventricular $(\mathrm{p}=0,046)$ level, after surgical repair. Three (33\%) patients had postoperative

\footnotetext{
* Correspondence: gabrielliguori@gmail.com

Heart Institute (InCor), University of Sao Paulo Medical School, Sao Paulo,
} Brazil

(c) 2013 Liguori et al; licensee BioMed Central Ltd. This is an Open Access article distributed under the terms of the Creative Commons and take full advantage of:

- Convenient online submission

- Thorough peer review

- No space constraints or color figure charges

- Immediate publication on acceptance

- Inclusion in PubMed, CAS, Scopus and Google Scholar

- Research which is freely available for redistribution

Submit your manuscript at 